\title{
Recurrent EZH1 mutations are a second hit in autonomous thyroid adenomas
}

\author{
Davide Calebiro, ${ }^{1,2}$ Elisa S. Grassi, ${ }^{3}$ Markus Eszlinger, ${ }^{4,5}$ Cristina L. Ronchi, ${ }^{6}$ Amod Codbole, ${ }^{1,2}$ Kerstin Bathon, ${ }^{1,2}$ \\ Fabiana Guizzardi, ${ }^{3}$ Tiziana de Filippis, ${ }^{3}$ Knut Krohn, ${ }^{7}$ Holger Jaeschke, ${ }^{5}$ Thomas Schwarzmayr, ${ }^{8,9}$ Rifat Bircan, ${ }^{10}$ \\ Hulya Iliksu Gozu, ${ }^{11}$ Seda Sancak, ${ }^{12}$ Marek Niedziela, ${ }^{13}$ Tim M. Strom, ${ }^{8,9}$ Martin Fassnacht,, 6,14 Luca Persani, ${ }^{3,15}$ and Ralf Paschke ${ }^{4}$ \\ IInstitute of Pharmacology and Toxicology, University of Würzburg, Würzburg, Germany. ${ }^{2}$ Bio-Imaging Center/Rudolf Virchow Center, Würzburg, Germany. ${ }^{3}$ Division of Endocrine and Metabolic Diseases \\ and Laboratory of Endocrine and Metabolic Research, Istituto Auxologico Italiano, Milan, Italy. ${ }^{4}$ Divisions of Endocrinology and Metabolism and Oncology and Arnie Charbonneau Cancer Research Institute, \\ University of Calgary, Calgary, Alberta, Canada. ${ }^{5}$ Division of Endocrinology and Nephrology, University of Leipzig, Leipzig, Cermany. ${ }^{6}$ Comprehensive Cancer Center Mainfranken, University of Würzburg, \\ Würzburg, Germany. ${ }^{7}$ IZKF Leipzig, University of Leipzig, Leipzig, Germany. ${ }^{8}$ Institute of Human Genetics, Helmholtz Zentrum München, Neuherberg, Germany. ${ }^{9}$ Institute of Human Genetics, Technische \\ Universität München, Munich, Germany. ${ }^{10}$ Department of Molecular Biology and Genetics, Namik Kemal University, Tekirdag, Turkey. "Department of Endocrinology and Metabolism, School of Medicine, \\ Pendik Education and Training Hospital, Marmara University, Istanbul, Turkey. ${ }^{2}$ Division of Endocrinology and Metabolism, Fatih Sultan Mehmet Training and Educational Hospital, Istanbul, Turkey. \\ ${ }^{13}$ Department of Paediatric Endocrinology and Rheumatology, Poznan University of Medical Sciences, Poznan, Poland. ${ }^{14}$ Department of Internal Medicine I, Division of Endocrinology and Diabetes, \\ University Hospital, University of Würzburg, Würzburg, Germany. ${ }^{15}$ Department of Clinical Sciences and Community Health, University of Milan, Milan, Italy.
}

\begin{abstract}
Autonomous thyroid adenomas (ATAs) are a frequent cause of hyperthyroidism. Mutations in the genes encoding the TSH receptor (TSHR) or the Gs protein $\alpha$ subunit (CNAS) are found in approximately $70 \%$ of ATAs. The involvement of other genes and the pathogenesis of the remaining cases are presently unknown. Here, we performed whole-exome sequencing in 19 ATAs that were paired with normal DNA samples and identified a recurrent hot-spot mutation (c.1712A>G; p.Gln571Arg) in the enhancer of zeste homolog 1 ( $E Z H 1$ ) gene, which codes for a catalytic subunit of the polycomb complex. Targeted screening in an independent cohort confirmed that this mutation occurs with high frequency (27\%) in ATAs. EZH1 mutations were strongly associated with known (TSHR, GNAS) or presumed (adenylate cyclase 9 [ADCY9]) alterations in cAMP pathway genes. Furthermore, functional studies revealed that the p.CIn571Arg EZH1 mutation caused increased histone $\mathrm{H} 3$ trimethylation and increased proliferation of thyroid cells. In summary, this study revealed that a hot-spot mutation in EZH1 is the second most frequent genetic alteration in ATAs. The association between EZH1 and TSHR mutations suggests a 2-hit model for the pathogenesis of these tumors, whereby constitutive activation of the CAMP pathway and EZH1 mutations cooperate to induce the hyperproliferation of thyroid cells.
\end{abstract}

\section{Introduction}

Autonomous thyroid adenomas (ATAs) are a major cause of hyperthyroidism, especially in iodine-deficient areas $(1,2)$. The thyroid-stimulating hormone (TSH) plays a major role in controlling the function and growth of thyroid cells. Binding of TSH to its receptor stimulates cAMP production and the subsequent activation of protein kinase A (PKA), leading to thyroid hormone production and cell proliferation. Consistently, activating mutations in the TSH receptor (TSHR) have been found in up to $70 \%$ of ATAs $(1,3,4)$. In addition, a minority of these tumors carry an activating mutation in the Gs protein $\alpha$ subunit (GNAS) gene (1, $3,4)$. While it is well established that constitutive activation of the cAMP/PKA pathway plays an important role in uncontrolled thyroid hormone secretion, it is presently unclear whether this is sufficient to induce the formation and/or expansion of thyroid adenomas. Moreover, the pathogenesis of a relevant fraction (approximately 30\%) of ATAs that do not harbor TSHR or GNAS mutations is presently unknown. Only a minor fraction of the

Conflict of interest: The authors have declared that no conflict of interest exists. Submitted: October 8, 2015; Accepted: June 16, 2016.

Reference information: / Clin Invest. 2016;126(9):3383-3388. doi:10.1172/JCI84894
TSHR and GNAS mutation-negative tumors identified in early studies may represent false negatives $(5,6)$.

Whole-exome sequencing of matched tumor and normal samples is a powerful method for identifying somatic mutations implicated in tumor development. This approach is particularly effective in the case of well-differentiated tumors, which harbor few mutations. Using this approach, the molecular pathogenesis of several endocrine diseases associated with hormone hypersecretion was recently clarified (7-9). Above all, this led to the identification of mutations in the catalytic $\alpha$ subunit of PKA (PRKACA) as being responsible for Cushing's syndrome due to cortisolsecreting adrenocortical adenomas (8).

In this study, we performed next-generation whole-exome sequencing in a large series of ATAs to investigate the genetic alterations underlying these tumors. We found that a relevant fraction of ATAs carry a recurrent hot-spot mutation in a key gene involved in the regulation of cell differentiation and proliferation.

\section{Results and Discussion}

By performing whole-exome sequencing in 19 ATAs and paired normal samples, we identified a total of 94 candidate somatic mutations (81 missense, 5 nonsense, 3 frameshift, 3 direct splic- 
A
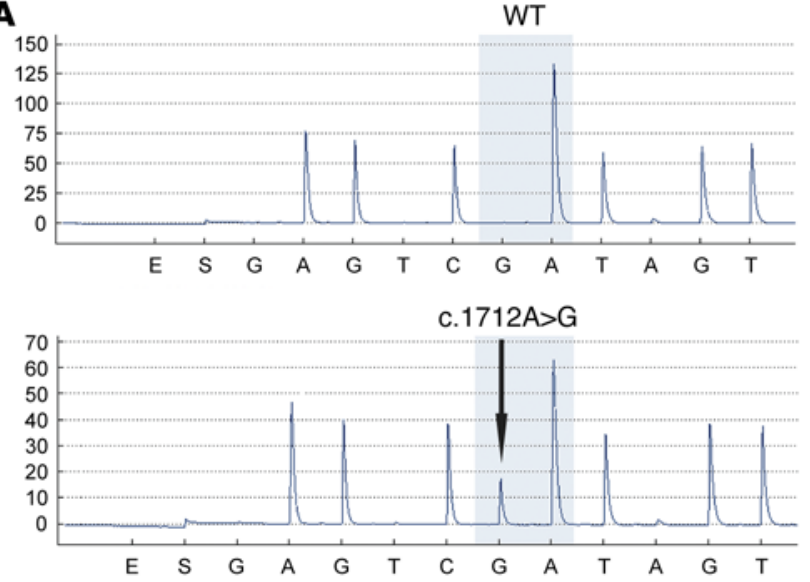

B

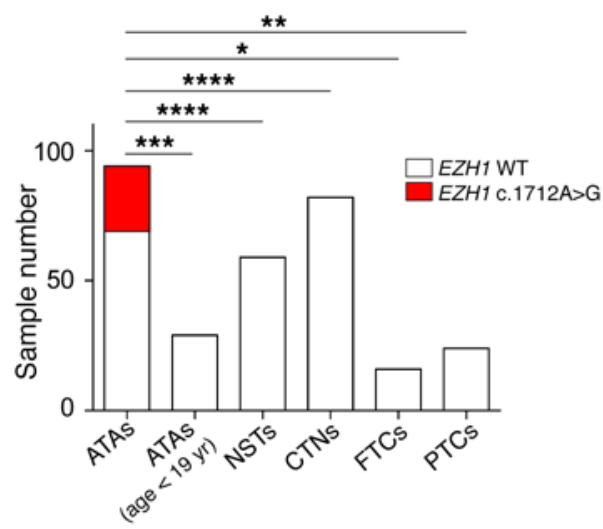

C

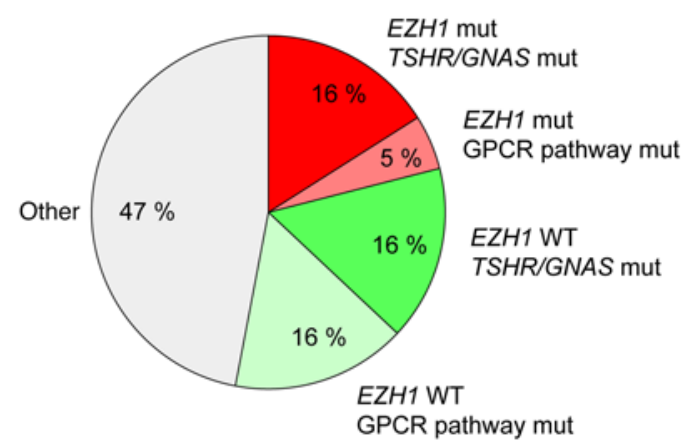

D

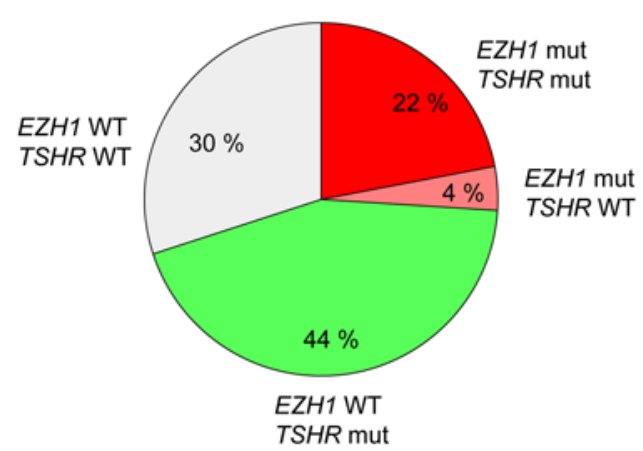

Figure 1. Identification of a hot-spot somatic EZH1 mutation in ATAs. (A) Sequence chromatogram of an ATA sample carrying the heterozygous c.1712A $>\mathrm{G}$ mutation. A WT sample is shown for comparison. (B) Frequency of the EZH1 c.1712A $>\mathrm{G}$ mutation in different types of thyroid nodules. ${ }^{*} P<0.05$, ${ }^{* *} P<0.01,{ }^{* *} P<0.001$, and ${ }^{* * *} P<0.0001$ by $\chi^{2}$ test. (C and $\left.\mathbf{D}\right)$ Grouping of the samples according to their mutational state in the exome-sequencing data (C) and validation cohort (D). mut, mutation.

ing, and 2 indel; median: 4 somatic mutations in exonic regions per ATA sample; range: 0-12; Supplemental Figure 1; supplemental material available online with this article; doi:10.1172/ JCI84894DS1). On the basis of a prediction of functional effects with the PolyPhen-2 software, 28 mutations were classified as probably damaging, 12 as possibly damaging, and 37 as benign. A detailed list of the observed somatic mutations is provided in Supplemental Table 1. These mutations affected a total of 84 genes, 78 of which were associated with a Gene Ontology (GO) term. A canonical pathway analysis (Supplemental Table 2) recognized the PDGF receptor pathway ( $n=5$ genes) and the GPCR pathway ( $n=9$ genes) as most significantly represented. The latter included 5 GPCRs (TSHR, galanin receptor 2 [GALR2], olfactory receptor family 2 subfamily $\mathrm{G}$ member 2 [OR2G2], olfactory receptor family 5 subfamily M member 3 [OR5M3], and taste 1 receptor member 2 [TAS1R2]; see also Appendix 1 and Supplemental Figure 2), the Gs protein a subunit (GNAS), adenylyl cyclase 9 (ADCY9), and the ribosomal protein S6 kinase (RPS6KA3). Mutations affecting 3 genes (enhancer of zeste homolog 1 [EZH1], thyroglobulin $[T G]$, and TSHR) were found in at least 2 samples and were therefore considered to be recurrent. In particular, a hotspot substitution (c.1712A $>$ G; p.Gln571Arg) in $E Z H 1$ was found in 4 tumors (21\% of cases) (Figure 1A). The c.1712A $>$ G substitution was not present in 6,951 in-house exomes nor in 60,706 exomes in the Exome Aggregation Consortium (ExAC) Browser (http:// exac.broadinstitute.org/; see Supplemental Methods for further details). This recurrent pattern of a heterozygous somatic mutation was highly suggestive of a gain-of-function mode of action. No correlation was found between the mutational status and the available clinical data (Supplemental Table 3), including age at surgery, tumor size, and thyroid function tests (TSH, FT4, and FT3 levels as well as AbTg and AbTPO positivity).

On the basis of these initial results, we screened a large series of thyroid nodule samples $(n=304)$ for the presence of the hot-spot EZH1 c.1712A $>\mathrm{G}$ mutation. These samples comprised 94 ATAs diagnosed in adults, 29 ATAs diagnosed in children and adolescents, 59 normal surrounding tissue samples (NSTs) of ATAs, 82 scintigraphically cold (i.e., nonfunctioning) thyroid nodules (CTNs), 16 follicular thyroid carcinomas (FTCs), and 24 papillary thyroid carcinomas (PTCs). Interestingly, the hot-spot EZH1 mutation was found in 25 of 94 (27\%) of the adult ATAs but not in any of the other samples (Figure 1B). A summary of the clinical parameters and mutational status of the adult ATA patients is presented in Supplemental Table 4. The absence of the hot-spot EZH1 mutation in FTCs and PTCs suggests that ATAs carrying this mutation are not a precursor to carcinoma. Further- 


\section{A}

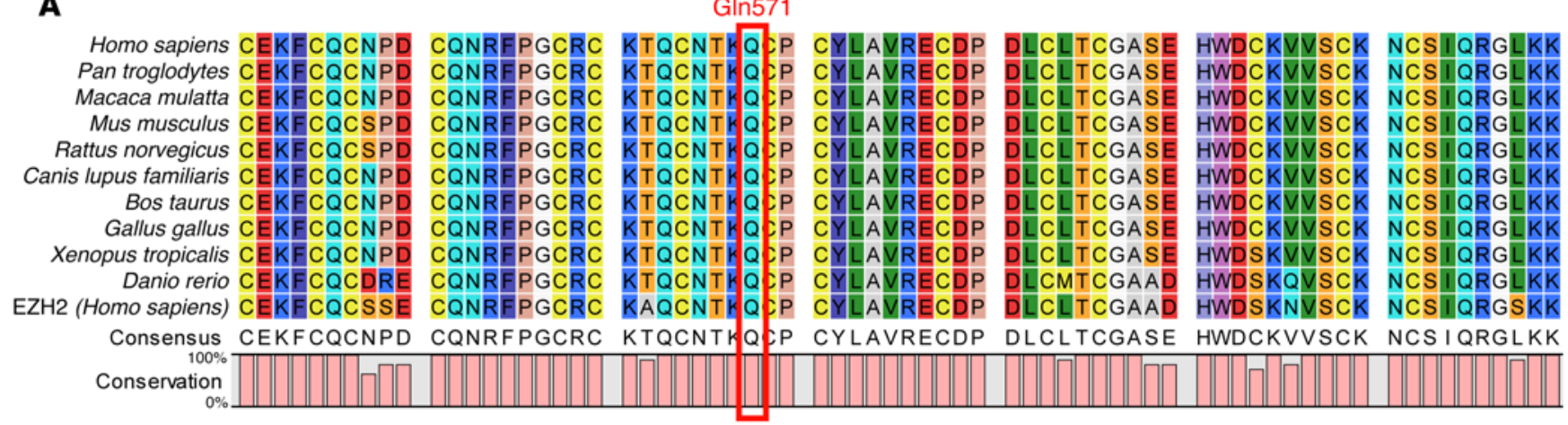

B

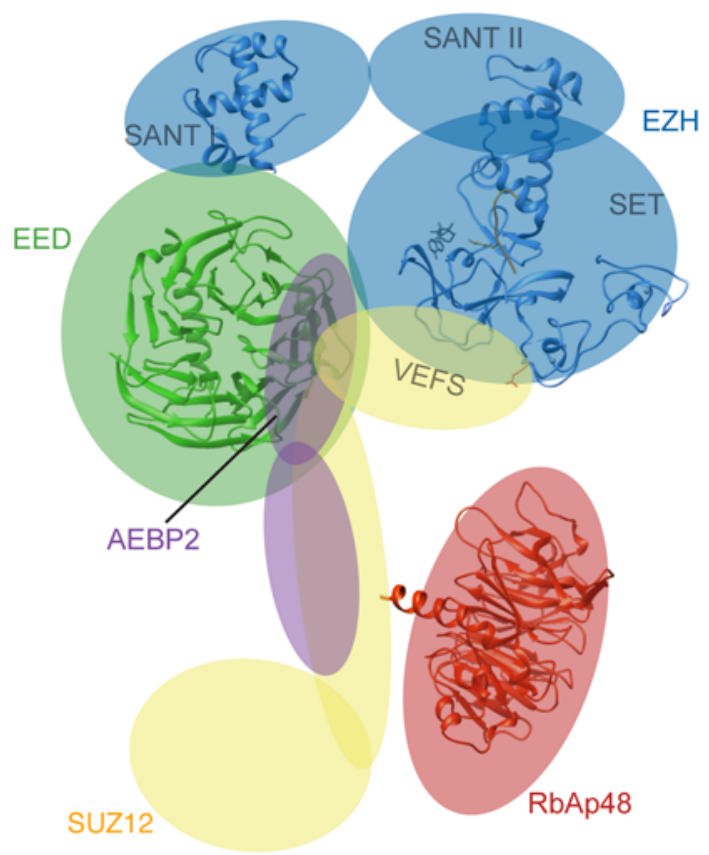

C

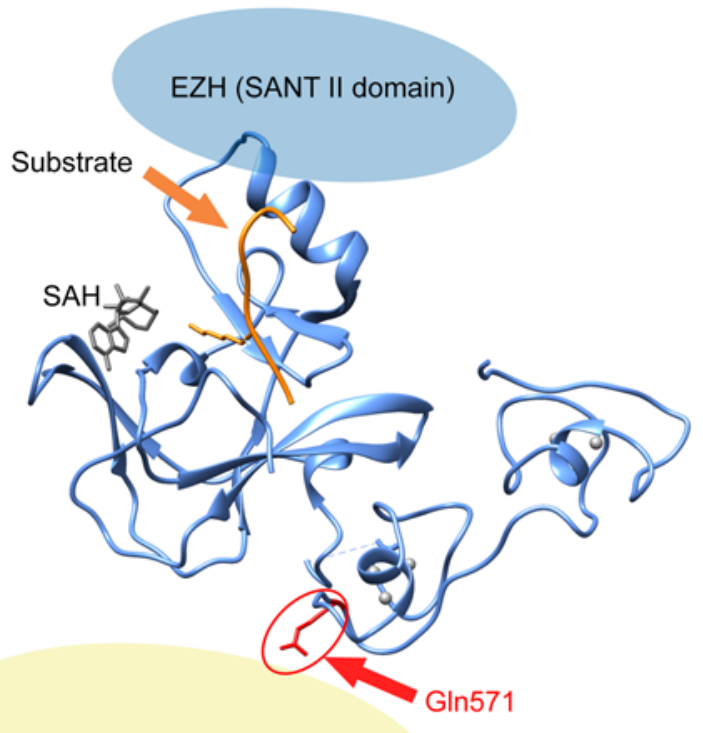

SUZ12 (VEFS domain)

Figure 2. Location of the GIn571Arg mutation in EZH1. (A) Sequence alignment of the region corresponding to GIn571 in EZH1 from different species. The sequence of the closely related human EZH2 is included. (B) Predicted overall structure of PRC2 (based on ref. 18). (C) Position of the residue corresponding to GIn571. Since no structural data are available for EZH1, a structure of the catalytic domain of EZH2 (Protein Data Bank [PDB] ID: 4MI5; $95 \%$ homology) is shown. The positions of the substrate and cofactor S-adenosyl-L-homocysteine (SAH) are based on an alignment with human H3K9 methyltransferase (PDB ID: 3HNA).

more, the lack of EZH1 mutations in pediatric ATAs suggests that these adenomas might represent a separate entity or that EZH1 mutations might be acquired later in the natural history of these tumors. No EZH1 mutations were found in either the thyroid tissue or peripheral leukocytes of members of a family with the rare condition of nonautoimmune hyperfunctioning thyroid hyperplasia associated with germline, activating TSHR mutations (10), nor were these mutations found in a 2-year-old female with a sporadic case. Remarkably, in the ATA group investigated by exome sequencing, EZH1 mutations were found only in samples carrying known mutations in other genes of the cAMP pathway, i.e., $\operatorname{TSHR}(n=2)$ and $\operatorname{GNAS}(n=1)$, or a potentially damaging mutation in ADCY9 $(n=1)$. Considering the known mutations affecting GPCR signaling (TSHR, GNAS), the hot-spot EZH1 mutation, and variations in other genes of the GPCR-signaling pathway, the ATAs analyzed by exome sequencing could be divided into 5 groups (Figure 1C). Similar results were obtained in the validation cohort when considering TSHR and EZH1 mutations (Figure 1D). In the validation cohort, approximately onethird (21 of 62, 34\%) of the TSHR mutation-positive ATAs harbored an $E Z H 1$ c. $1712 A>G$ mutation. Similar allelic percentages in a subgroup of 8 ATAs suggested a possible clonal origin of the cells with both mutations (Supplemental Figure 3).

EZH1 codes for a catalytic subunit of the polycomb repressive complex 2 (PRC2), which is implicated in the maintenance of embryonic stem cell pluripotency and plasticity. EZH1, a SET domain-containing histone methyltransferase, exerts its effects by catalyzing the mono-, di- and trimethylation of histone $\mathrm{H} 3$ at Lys27, leading to transcriptional repression of the associated genes $(11,12)$. PRC2 has been linked to the aggressiveness of various cancers $(13,14)$, and selective EZH1/2 inhibitors have been proposed as a novel anticancer strategy (15). 

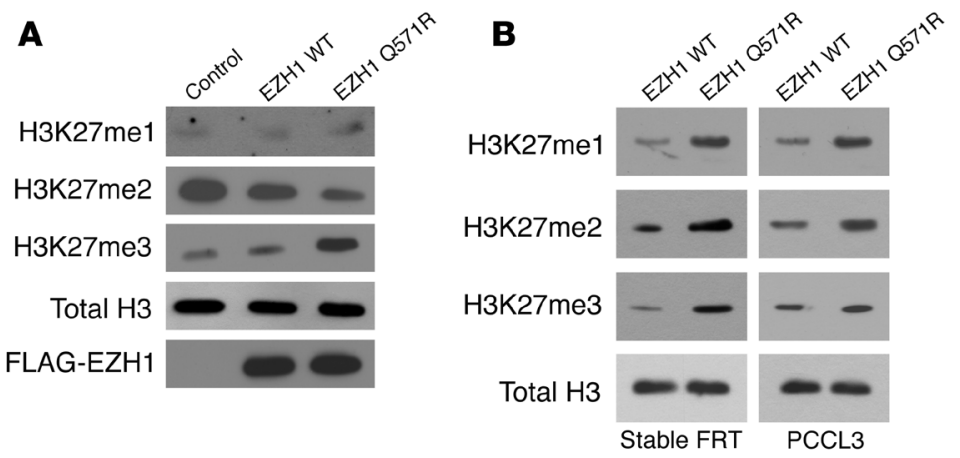

C NST
ATA

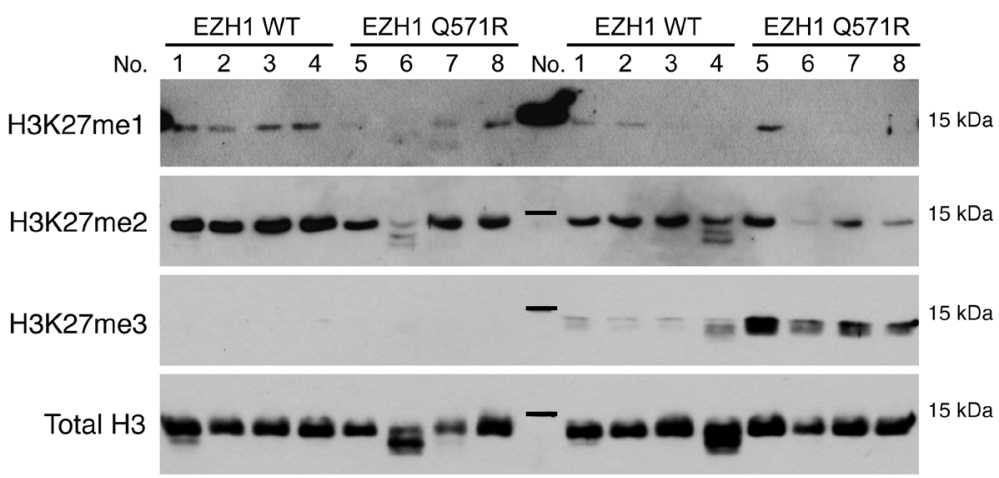

D

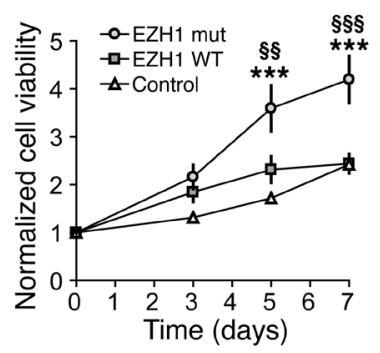

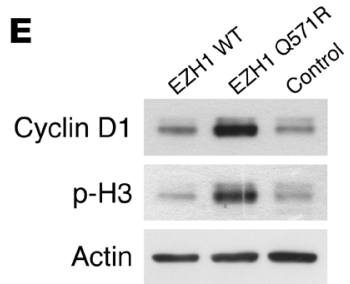

Figure 3. Functional characterization of the GIn571Arg EZH1 mutant. (A) Effect of EZH1 GIn571Arg on histone H3 methylation at Lys27. The methylation pattern in lysates from cells transfected with FLAG-tagged EZH1 WT/GIn571Arg or the empty expression vector (control) was evaluated by Western blot analysis with Abs against mono- (H3K27me1), di- (H3K27me2), and trimethylation (H3K27me3). The expression of transfected EZH1 was evaluated using an Ab against the FLAC tag. (B) H3K27 methylation in thyroid cell lines stably (FRT) or transiently (PCCL3) transfected with EZH1 WT/GIn571Arg. (C) H3K27 methylation in ATA samples with or without GIn571Arg EZH1 mutation and the corresponding NSTs. (D) Proliferation of FRT cells stably expressing EZH1 WT/GIn571Arg. Cell viability was measured with the MTT assay. Data $(n=8)$ are shown as the mean \pm SEM. ${ }^{* * *} P<0.001$ versus control; ${ }^{\circledR} P<0.01$ versus EZH1 WT; $\$ \S \S P<0.001$ versus EZH1 WT. Statistical significance was determined by 2-way ANOVA, followed by Bonferroni's post test (E) Western blot analysis of proliferative markers in FRT cells stably expressing EZH1 WT/GIn571Arg. Results in A, $\mathbf{B}$, and $\mathbf{E}$ are representative of 3 independent experiments. p-H3, phosphorylated H3.
The amino acid change (Gln571Arg) identified in ATAs in this study involves a highly conserved amino acid in the catalytic (SET) domain of EZH1 (Figure 2A). The position of this residue in a PRC2 model based on the partially solved x-ray structure of the closely related homolog EZH2 (14) is shown in Figure 2, B and C. This amino acid immediately precedes a cysteine residue, which is directly involved in the coordination of a zinc atom in the second zinc-binding domain (14).

Given these findings, we investigated whether the Gln571Arg mutation was affecting EZH1 activity in intact cells. For this purpose, we transfected HEK293 cells with either WT or mutant EZH1 and evaluated histone $\mathrm{H} 3$ methylation using Abs specific to mono-, di-, or trimethylated Lys27. Expression levels of both WT and mutant EZH1 protein were similar, indicating that the Gln571Arg mutation was not affecting protein stability (Figure 3A). Remarkably, expression of the Gln571Arg mutant caused a strong increase in histone $\mathrm{H} 3$ trimethylation, accompanied by a concomitant decrease in dimethylation (Figure 3A). Moreover, an increase in histone $\mathrm{H} 3$ methylation was observed upon expression of the Gln571Arg mutant in 2 rat thyroid cell models: transiently transfected PCCL3 and stably transfected Fischer rat thyroid (FRT) cells (Figure 3B). In addition, we analyzed histone
$\mathrm{H} 3$ methylation in a subset of ATA samples with or without the EZH1 mutation. All analyzed ATAs with EZH1 mutation had higher trimethylation levels and a tendency toward lower dimethylation levels compared with those detected in both the corresponding NST samples and the EZH1 mutation-negative ATAs (Figure $3 \mathrm{C}$ ). The increase in histone $\mathrm{H} 3$ trimethylation is similar to that found with EZH2 mutations in follicular and diffuse large B cell lymphomas (16). However, the mechanism is probably different. The EZH2 mutations in B cell lymphomas affect residues (Y641 and Y677) that are directly involved in substrate binding (14), thus altering the substrate specificity in favor of dimethylated histone $\mathrm{H} 3$ and ultimately leading to increased Lys27 trimethylation (17). The EZH1 mutation found in our study (Gln571Arg) affects a solvent-exposed residue in a region that is highly conserved between EZH1 and EZH2. Given the information available for $\mathrm{EZH} 2$, this residue is probably not involved in substrate binding but might play a role in the interaction with the suppressor of zeste 12 homolog (SUZ12) subunit of PRC2 and the cofactor adipocyte enhancer-binding protein 2 (AEBP2), which is required for optimal enzymatic activity $(18,19)$ (Figure 2 , B and C). Thus, we investigated the interaction between the Gln571Arg mutant and PRC2 components (SUZ12, AEBP2, and embryonic ectoderm 
development [EED]) by coimmunoprecipitation (Supplemental Figure 4). No differences were detected between WT and mutant EZH1 proteins, suggesting that the Gln571Arg mutation does not grossly alter these interactions. Detailed structural studies will be required to further dissect the mechanisms underlying the increased activity caused by the Gln571Arg mutation.

Finally, we evaluated whether the EZH1 Gln571Arg substitution was affecting the proliferation of thyroid cells. For this purpose, we generated FRT cells stably expressing WT EZH1 or the Gln571Arg mutant. Stable expression of the Gln571Arg mutant was associated with increased cell proliferation (Figure 3D). This effect was accompanied by a strong increase in the levels of cyclin D1 and histone H3 phosphorylation at Ser10, two cell proliferation markers (Figure 3E). In addition, we compared the effects of transient EZH1 transfection in 2 FRT clones stably expressing the WT TSHR or an activating TSHR mutant (V597F) (20). In contrast to transfection of WT EZH1, which had only a moderate proliferative effect, transfection of the mutant EZH1 caused a strong increase of cell proliferation in both cell lines (Supplemental Figure 5). These data suggest that the Gln571Arg mutant has a relevant impact on the proliferation of thyroid cells and can increase cell proliferation also in the presence of an activating TSHR mutant.

In conclusion, this study identifies EZH1 mutations as the second most frequent genetic alteration in ATAs. Gain-of-function TSHR mutations may well be sufficient to support thyroid autonomy and hyperplasia, as thyroid-stimulating Abs (TSAbs) do in Graves' disease. However, we propose that their association with EZH1 mutations such as those found in a relevant subset of ATAs in this study - or with other still-unknown events giving advantage over normal thyroid cells - might further promote adenoma growth according to a "2-hit" model. Testing for the EZH1 Gln571Arg hot-spot mutation might help in the molecular diagnosis and classification of thyroid nodules. Moreover, interfering with $E Z H 1$ function might be of potential therapeutic benefit in thyroid disorders associated with increased cell proliferation. Additional mutations and/or pathogenic mechanisms remain to be identified for cases that are both TSHR and EZH1 mutation negative.

\section{Methods}

Further information can be found in the Supplemental Methods. See the complete unedited blots in the supplemental material.
Statistics. Statistical analyses were performed using GraphPad Prism 4.0 software (GraphPad Software). The Fisher's exact or $\chi^{2}$ test was used to investigate dichotomic variables. Differences between 3 or more groups were assessed by 1-way or 2-way ANOVA. P values of less than 0.05 were considered statistically significant.

Study approval. Patients were recruited at the University of Leipzig, the Istituto Auxologico Italiano, the Poznan University of Medical Sciences, and the School of Medicine of Marmara University. All patients provided written informed consent, and the study was approved by the ethics committee of each participating institution.

\section{Author contributions}

DC, TS, MF, LP, and RP designed the study. ESG, ME, CLR, AG, $\mathrm{KB}, \mathrm{FG}, \mathrm{TdF}, \mathrm{KK}$, and $\mathrm{HJ}$ performed experiments and analyzed data. RB, HIG, SS, and MN collected patients' samples and clinical data. TMS performed whole-exome sequencing. DC, LP, and RP wrote the manuscript.

\section{Acknowledgments}

We wish to thank Eileen Bösenberg, Bianca Klüpfel, and Ines Elsner for technical support and Ulrike Zabel for DNA cloning. This study was partially supported by grants from the IZKF Würzburg (B-281, to DC and MF); the ERA-NET "E-Rare" (O1GM1407B, to MF and DC); the Deutsche Krebshilfe (109994, to ME); and the Wilhelm Sander Stiftung (project 2013.010.1, to RP).

Address correspondence to: Davide Calebiro, Institute of Pharmacology and Toxicology and Bio-Imaging Center/Rudolf Virchow Center, University of Würzburg, Versbacher Str. 9, 97078 Würzburg, Germany. Phone: 49.931.31.80067; E-mail: davide.calebiro@ toxi.uni-wuerzburg.de. Or to: Luca Persani, University of Milan, Division of Endocrinology and Metabolism, IRCCS Istituto Auxologico Italiano, Piazzale Brescia 20, 20149 Milan, Italy. Phone: 39.02.61911.2400; E-mail: luca.persani@unimi.it. Or to: Ralf Paschke, University of Calgary, Division of Endocrinology and Metabolism, Arnie Charbonneau Cancer Research Institute and Alberta Health Services - Calgary Zone, Richmond Road Diagnosis and Treatment Centre (RRDTC), 1820 Richmond RD SW, Calgary AB T2T5C7. Phone: 403.955.8959; E-mail: ralf.paschke@ucalgary.ca.

HJ's present address is: Institute of Biomedicine, Department of Physiology, University of Turku, Turku, Finland.
1. Krohn K, Paschke R. Somatic mutations in thyroid nodular disease. Mol Genet Metab. 2002;75(3):202-208.

2. Kopp P. Thyrotoxicosis of other etiologies. In: De Groot LJ, et al. eds. Endotext. South Dartmouth, MA; 2000.

3. Parma J, et al. Somatic mutations in the thyrotropin receptor gene cause hyperfunctioning thyroid adenomas. Nature. 1993;365(6447):649-651.

4. Persani L, et al. Induction of specific phosphodiesterase isoforms by constitutive activation of the cAMP pathway in autonomous thyroid adenomas. JClin Endocrinol Metab. 2000;85(8):2872-2878.

5. Trülzsch B, Krohn K, Wonerow P, Paschke R. DGGE is more sensitive for the detection of somatic point mutations than direct sequencing.
BioTechniques. 1999;27(2):266-268.

6. Eszlinger M, et al. Somatic mutations in 33 benign and malignant hot thyroid nodules in children and adolescents. Mol Cell Endocrinol. 2014;393(1-2):39-45.

7. Beuschlein F, et al. Somatic mutations in ATP1A1 and ATP2B3 lead to aldosterone-producing adenomas and secondary hypertension. Nat Genet. 2013;45(4):440-444e1.

8. Beuschlein F, et al. Constitutive activation of PKA catalytic subunit in adrenal Cushing's syndrome. N Engl J Med. 2014;370(11):1019-1028.

9. Reincke M, et al. Mutations in the deubiquitinase gene USP8 cause Cushing's disease. Nat Genet. 2015;47(1):31-38.

10. Führer D, Wonerow $\mathrm{P}$, Willgerodt $\mathrm{H}$, Paschke
R. Identification of a new thyrotropin receptor germline mutation (Leu629Phe) in a family with neonatal onset of autosomal dominant nonautoimmune hyperthyroidism. J Clin Endocrinol Metab. 1997;82(12):4234-4238.

11. Shen X, et al. EZH1 mediates methylation on histone H3 lysine 27 and complements EZH2 in maintaining stem cell identity and executing pluripotency. Mol Cell. 2008;32(4):491-502.

12. Margueron R, et al. Ezh1 and Ezh2 maintain repressive chromatin through different mechanisms. Mol Cell. 2008;32(4):503-518.

13. Valk-Lingbeek ME, Bruggeman SW, van Lohuizen M. Stem cells and cancer; the polycomb connection. Cell. 2004;118(4):409-418.

14. Antonysamy S, et al. Structural context of dis- 
ease-associated mutations and putative mechanism of autoinhibition revealed by X-ray crystallographic analysis of the EZH2-SET domain. PLOS ONE. 2013;8(12):e84147.

15. Xu B, Konze KD, Jin J, Wang GG. Targeting EZH2 and PRC2 dependence as novel anticancer therapy. Exp Hematol. 2015;43(8):698-712.

16. Morin RD, et al. Somatic mutations altering EZH2 (Tyr641) in follicular and diffuse large
B-cell lymphomas of germinal-center origin. Nat Genet. 2010;42(2):181-185.

17. Yap DB, et al. Somatic mutations at EZH2 Y641 act dominantly through a mechanism of selectively altered PRC2 catalytic activity, to increase H3K27 trimethylation. Blood. 2011;117(8):2451-2459.

18. Ciferri C, Lander GC, Maiolica A, Herzog F, Aebersold R, Nogales E. Molecular architecture of human polycomb repressive complex 2. Elife. 2012;1:e00005.

19. Cao R, Zhang Y. SUZ12 is required for both the histone methyltransferase activity and the silencing function of the EED-EZH2 complex. Mol Cell. 2004;15(1):57-67.

20. Alberti L, et al. A novel germline mutation in the TSH receptor gene causes non-autoimmune autosomal dominant hyperthyroidism. Eur $J$ Endocrinol. 2001;145(3):249-254. 